\title{
Fabry Disease: Thirty-Five Mutations in the $\alpha$-Galactosidase A Gene in Patients with Classic and Variant Phenotypes
}

\author{
Christine M. Eng, *,t Grace A. Ashley,* Tania S. Burgert,* \\ Annette L. Enriquez,* Marcus D'Souza,* and \\ Robert J. Desnick*'† \\ Departments of ${ }^{*}$ Human Genetics and ${ }^{\dagger}$ Pediatrics, Mount Sinai School \\ of Medicine, New York, New York, U.S.A.
}

\begin{abstract}
Background: Fabry disease, an X-linked inborn error of glycosphingolipid catabolism, results from mutations in the $\alpha$-galactosidase $\mathrm{A}(\alpha$-Gal A) gene located at Xq22.1. To determine the nature and frequency of the molecular lesions causing the classical and milder variant Fabry phenotypes and for precise carrier detection, the $\alpha$-Gal A lesions in 42 unrelated Fabry hemizygotes were determined.

Materials and Methods: Genomic DNA was isolated from affected probands and their family members. The seven $\alpha$-galactosidase A exons and flanking intronic sequences were PCR amplified and the nucleotide sequence was determined by solid-phase direct sequencing.

Results: Two patients with the mild cardiac phenotype had missense mutations, I91T and F113L, respectively. In 38 classically affected patients, 33 new mutations
\end{abstract}

were identified including 20 missense $(\mathrm{MlT}, \mathrm{A} 31 \mathrm{~V}$, H46R, Y86C, L89P, D92Y, C94Y, A97V, R100T, Y134S, G138R, A143T, S148R, G163V, D170V, C202Y, Y216D, N263S, W287C, and N298S), two nonsense (Q386X, W399X), one splice site mutation (IVS4 $+2 \mathrm{~T} \rightarrow \mathrm{C}$ ), and eight small exonic insertions or deletions (304dell, 613del9, 777del1, 1057del2, 1074del2, 1077dell, 1212del3, and 1094ins1), which identified exon 7 as a region prone to gene rearrangements. In addition, two unique complex rearrangements consisting of contiguous small insertions and deletions were found in exons 1 and 2 causing L45R/H46S and L120X, respectively.

Conclusions: These studies further define the heterogeneity of mutations causing Fabry disease, permit precise carrier identification and prenatal diagnosis in these families, and facilitate the identification of candidates for enzyme replacement therapy.

\section{INTRODUCTION}

Fabry disease is an X-linked inborn error of glycosphingolipid catabolism resulting from the deficient activity of the lysosomal exoglycohydrolase, $\alpha$-galactosidase A (EC 3.2.1.22; $\alpha$-Gal A) (1). Affected hemizygous males accumulate neutral glycosphingolipids with terminal $\alpha$-linked galactosyl moieties particularly in the plasma and in lysosomes of blood vessels, heart, liver, and kidney. In classically affected males-who have

Address correspondence and reprint requests to: C. M. Eng, Department of Human Genetics, Box 1498, One Gustave Levy Place, New York, NY 10029, U.S.A. Phone: 212-2416944; Fax: 212-360-1809. little, if any, detectable $\alpha$-Gal A activity-the onset of disease manifestations occurs in childhood or adolescence and is characterized by severe acroparesthesias, angiokeratoma, corneal opacities, and hypohidrosis. With advancing age, vascular disease of the heart, kidneys, and brain lead to early demise in adulthood. Asymptomatic or clinically milder hemizygotes with residual $\alpha$-Gal A activity have an attenuated form of the disease limited to cardiac involvement (the "cardiac variants"), including cardiomegaly, typically involving the left ventricular wall and interventricular septum, EKG changes consistent with a cardiomyopathy, and myocardial infarctions (1). The disease has a panethnic distribution with an es- 
timated frequency of 1 in 40,000 males. However, the frequency may be underestimated because of a lack of ascertainment and failure to diagnose mild or atypical cases (2).

Our understanding of human $\alpha$-Gal $A$ and Fabry disease advanced markedly with the isolation and characterization of the full-length cDNA and the genomic sequences for this lysosomal hydrolase (3-6). The cDNA encodes a polypeptide of 429 residues, including a 31-amino acid signal peptide. The $12-\mathrm{kb} \alpha-\mathrm{Gal}$ A gene contains seven exons (6) and is one of the most Alu-rich genes with about $1 \mathrm{Alu} / \mathrm{kb}$. The gene was localized to the long arm of the $\mathrm{X}$ chromosome within band Xq22 by in situ hybridization (1). The Xq22 region spans approximately $10-12 \mathrm{Mb}$ of the proximal long arm of the $\mathrm{X}$ chromosome and also contains the $\alpha 5$ (IV) collagen gene (7) that is defective in Alport syndrome (8) and the agammaglobulinemia tyrosine kinase gene that is defective in Bruton's agammaglobulinemia (9). Simple sequence repeat polymorphisms located in this region have been useful for carrier detection and prenatal diagnosis of Fabry disease by linkage analysis in informative families (10).

A variety of mutations in the $\alpha$-Gal A gene causing Fabry disease have been described, including partial gene rearrangements, small deletions and insertions, splice-junction consensus site alterations, and various coding region single base substitutions (11). Most mutations resulted in the classic disease phenotype; however, five missense mutations were detected in hemizygotes with the mild cardiac variant phenotype (12-14). To date, most mutations have been confined to a single family with the exception of mutations involving CpG dinucleotides (R227Q, R227X, R342Q, and R342X), which were each found in two to six (R227X) unrelated classically affected families (14), and N215S, which was described in three unrelated cardiac variants (15).

To identify genotype/phenotype correlations, permit precise heterozygote detection, and facilitate selection of potential candidates for recombinant enzyme replacement trials, the nature and frequency of the $\alpha$-Gal A mutations causing Fabry disease in 42 unrelated families were determined. In this study, 35 new mutations are described, including missense, nonsense, and splice-site mutations as well as small and large gene rearrangements, emphasizing the genetic heterogeneity of this disease.
MATERIALS AND METHODS

Establishment of Lymphoblasts and Isolation of Genomic DNA

Blood samples collected in EDTA or acid-citrate dextrose (ACD) were obtained from affected hemizygotes, heterozygous females, and other family members with informed consent. The enzymatic diagnosis of affected hemizygotes and heterozygotes was established by determination of the 4-methylumbelliferyl- $\alpha$-galactosidase A activity in plasma, leukocytes, cultured fibroblasts, or lymphoblasts (16). The phenotypic manifestations of each patient were evaluated by the authors (C.M.E. and R.J.D.). Lymphoblast lines were established and maintained as previously described (17). Genomic DNA was isolated from whole blood or lymphoblasts by standard techniques (18).

\section{Identification of Gene Rearrangements}

Initial screening for major gene rearrangements (>30 bp) was accomplished by multiplex polymerase chain reaction (PCR) amplification of $\alpha$-Gal A sequences according to the method of Kornreich and Desnick (19).

\section{Genomic Amplification and Single-Stranded Solid-Phase Direct Sequencing}

$\alpha$-Gal A exons and their flanking intronic sequences were amplified from genomic DNA using primer sets containing one biotinylated primer as previously described (15). The biotinylated, single-stranded amplified products were sequenced by direct solid-phase dideoxy chain sequencing. In each proband, the entire coding sequence and all flanking intronic sequences were determined.

\section{RESULTS}

Multiplex (PCR) amplification of the $\alpha$-Gal A sequences did not reveal major gene deletions in genomic DNA from any of the 40 unrelated hemizygotes studied. However, by PCR-amplification and solid-phase direct sequencing, 35 new mutations were detected, including 22 missense (MlT, A31V, H46R, Y86C, L89P, I91T, D92Y, C94Y, A97V, R100T, F113L, Y134S, G138R, Al43T, S148R, G163V, D170V, C202Y, Y216D, N263S, W287C, and N298S); two nonsense 
TABLE 1. $\alpha$-Galactosidase A mutations causing Fabry disease

\begin{tabular}{|c|c|c|c|}
\hline Location & $\begin{array}{l}\text { Allele Designation } \\
\text { cDNA-Based }^{a}\end{array}$ & $\begin{array}{c}\text { Genomic Nucleotide } \\
\text { Change }^{b}\end{array}$ & Phenotype \\
\hline \multicolumn{4}{|l|}{ Missense } \\
\hline EX 1 & MlT & $\mathrm{gl} 181 \mathrm{~T} \rightarrow \mathrm{G}$ & Classic \\
\hline EX l & A31V & $\mathrm{g} 1271 \mathrm{C} \rightarrow \mathrm{T}$ & Classic \\
\hline EX l & H46R & $\mathrm{gl} 316 \mathrm{~A} \rightarrow \mathrm{G}$ & Classic \\
\hline EX 2 & Y86C & g5156A $\rightarrow$ G & Classic \\
\hline EX 2 & L89P & g5165T $\rightarrow C$ & Classic \\
\hline EX 2 & I91T & g5171T $\rightarrow C$ & Cardiac variant \\
\hline EX 2 & D92Y & g5173G $\rightarrow A$ & Classic \\
\hline EX 2 & C94Y & g5180G $\rightarrow A$ & Classic \\
\hline EX 2 & A97V & g5189C $\rightarrow \mathrm{T}$ & Mild \\
\hline EX 2 & R100T & g5198G $\rightarrow A$ & Classic \\
\hline EX 2 & F113L & g5236T $\rightarrow C$ & Cardiac variant \\
\hline EX 3 & Y134S & $\mathrm{g} 7300 \mathrm{~A} \rightarrow \mathrm{C}$ & Classic \\
\hline EX 3 & G138R & $\mathrm{g} 7311 \mathrm{G} \rightarrow \mathrm{A}$ & Classic \\
\hline EX 3 & $\mathrm{Al} 43 \mathrm{~T}$ & $\mathrm{~g} 7326 \mathrm{G} \rightarrow \mathrm{A}$ & Unknown $^{c}$ \\
\hline EX 3 & Sl48R & $\mathrm{g} 7343 \mathrm{~T} \rightarrow \mathrm{G}$ & Classic \\
\hline EX 3 & Gl63V & $\mathrm{g} 7387 \mathrm{G} \rightarrow \mathrm{T}$ & Classic \\
\hline EX 3 & Dl70V & $\mathrm{g} 7408 \mathrm{~A} \rightarrow \mathrm{T}$ & Classic \\
\hline EX 4 & C202Y & $\mathrm{g} 8378 \mathrm{G} \rightarrow \mathrm{A}$ & Classic \\
\hline EX 5 & Y216D & $\mathrm{g} 10137 \mathrm{~T} \rightarrow \mathrm{G}$ & Classic \\
\hline EX 5 & N263S & $\mathrm{gl} 10279 \mathrm{~A} \rightarrow \mathrm{G}$ & Classic \\
\hline EX 6 & W287C & $\mathrm{g} 10568 \mathrm{G} \rightarrow \mathrm{T}$ & Classic \\
\hline EX 6 & N298S & $\mathrm{gl} 10601 \mathrm{~A} \rightarrow \mathrm{G}$ & Classic \\
\hline \multicolumn{4}{|l|}{ Nonsense } \\
\hline EX 7 & Q386X & $\mathrm{gl1134C} \rightarrow \mathrm{T}$ & Classic \\
\hline EX 7 & W399X & g11174G $\rightarrow \mathrm{A}$ & Classic \\
\hline \multicolumn{4}{|l|}{ Splicing } \\
\hline Intron 4 & IVS4 $+2 \mathrm{~T} \rightarrow \mathrm{C}$ & g8414T $\rightarrow C$ & Classic \\
\hline \multicolumn{4}{|c|}{ Rearrangements } \\
\hline EX 2 & 304dell & 5204 delC & Classic \\
\hline EX 4 & 613 del9 & 8386del9 & Classic \\
\hline EX 5 & 777dell & 10268delA & Classic \\
\hline EX 7 & $1057 \mathrm{del} 2$ & 11035 delAT & Classic \\
\hline EX 7 & 1074del2 & 11053 delGA & Classic \\
\hline EX 7 & 1077del1 & 11055 delT & Classic \\
\hline EX 7 & $1212 \mathrm{del} 3$ & R404del & Classic \\
\hline EX 7 & 1094ins 1 & $11072 \mathrm{insC}$ & Classic \\
\hline \multicolumn{4}{|l|}{ Complex } \\
\hline EX 1 & & g1312TGCAC $\rightarrow$ GCTCG & Classic \\
\hline EX 2 & & g5115GGCAGAGCTCATG $\rightarrow$ GCAGAGCCA & Classic \\
\hline
\end{tabular}




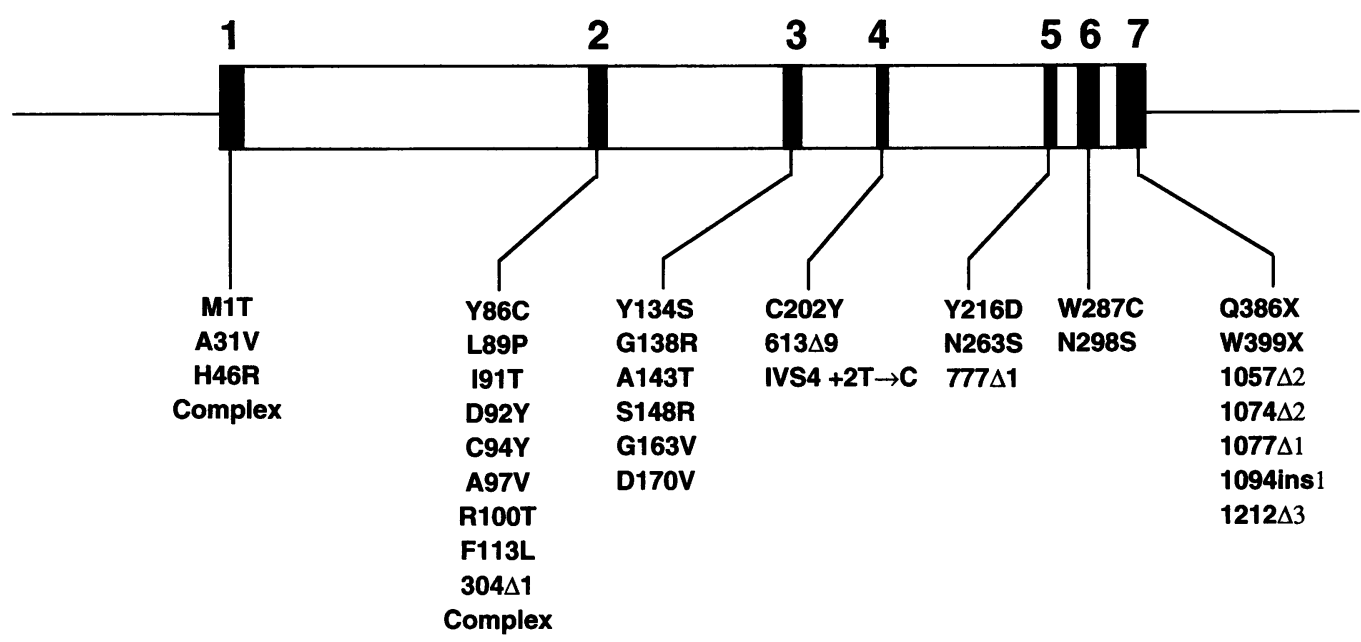

FIG. 1. Schematic of the $\alpha$-Gal A gene indicating the relative position of the seven exons and listing the mutations identified in each

(Q386X and W399X); one splice site mutation $(\mathrm{IVS} 4+2 \mathrm{~T} \rightarrow \mathrm{C})$; eight small gene rearrangements (304del1, 613del9, 777dell, 1057del2, 1074del2, 1077del1, 1212del3, and 1094ins1); and two novel complex insertion/deletion mutations in exons 1 and 2 (Table 1). Three of these point mutations (A31V, A97V, and H46R) occurred at $\mathrm{CpG}$ dinucleotides and two missense mutations were identified in mildly affected cardiac variants. In seven classically affected families, previously identified mutations were detected, including R49S, P205T, W226X, D313Y, and R227X, as well as Q157X, which occurred in two families.

\section{$\alpha$-Gal A Missense and Nonsense Mutations}

Of the 24 coding region point mutations found in the $\alpha$-Gal A gene, 22 were missense mutations and two were nonsense mutations (Fig. 1). The missense mutations detected in classically affected male patients included: (1) a T-to-G transversion in codon 1 , predicting a methionine-tothreonine substitution (MIT); (2) a C-to-T transition at a $\mathrm{CpG}$ dinucleotide in codon 31, resulting in an alanine-to-valine replacement (A31V); (3) an A-to-G transition at a CpG dinucleotide in codon 46 , resulting in a histidine-toarginine change (H46R); (4) an A-to-G transition in codon 86, predicting a tyrosine-to-cysteine substitution (Y86C); (5) a T-to-C transition in codon 89, predicting a leucine-to-proline replacement (L89P); (6) a T-to-C transition in codon 91, resulting in an isoleucine-to-threonine substitution (I91T); (7) a G-to-A transition in codon 92, predicting an aspartic acid-to-tyrosine change (D92Y); (8) a G-to-A transition in codon 94, predicting an cysteine-to-tyrosine replacement (C94Y); (9) a C-to-T transition in codon 97, resulting in an arginine-to-valine replacement (A97V); (10) a G-to-C transversion in codon 100, resulting in an arginine-to-threonine substitution (R100T); (11) a T-to-C transition in codon 113, predicting a phenylalanine-to-leucine replacement (F1 13L); (12) an A-to-C transversion in codon 134, predicting a tyrosine-to-serine substitution (Y134S); (13) a G-to-A transition in codon 138 , predicting a glycine-to-arginine replacement (G138R); (14) a G-to-A transition in codon 143, resulting in an alanine-to-threonine substitution (A143T); (15) a T-to-G transversion in codon 148, predicting a serine-to-arginine replacement (S148R); (16) a G-to-T transversion in codon 163, resulting in a glycine-to-valine substitution (G163V); (17) an A-to-T transversion in codon 170 , resulting in an aspartic acid-to-valine replacement (D170V); (18) a G-to-A transition in codon 202 , predicting a cysteine-to-tyrosine substitution (C202Y); (19) a T-to-G transversion in codon 216, resulting in a tyrosine-to-aspartic acid substitution (Y216D); (20) an A-to-G transition in codon 263, resulting in an asparagineto-serine replacement (N263S); (21) a G-to-T transversion in codon 287, predicting a typtophan-to-cysteine substitution (W287C); and (22) an A-to-G transition in codon 298, resulting in 
an asparagine-to-serine replacement (N298S). The nonsense mutations included (1) a C-to-T transition in codon 386, resulting in a glutamine to stop (Q386X), and (2) a G-to-A transition in codon 399, predicting a tryptophan to stop (W399X). Of note is that both nonsense mutations occurred in exon 7 at the carboxy-end of the $\alpha$-Gal A polypeptide.

\section{$\alpha-$ Gal A Splice-Site Mutation}

One splice site single-base alteration (gt $\rightarrow \mathrm{gc}$ ) was identified at the $5^{\prime}$ donor consensus splice site of intron 4 (designated IVS4+2T $\rightarrow$ C), which would be predicted to result in aberrant mRNA processing.

\section{$\alpha$-Gal A Gene Rearrangements}

Eight coding-region small gene rearrangements were identified, including five deletions of one or two bases (304dell, 777dell, 1057del2, 1074del2, and $1077 \mathrm{dell}$ ) that resulted in frameshift mutations and premature termination of the polypeptide chain; two in-frame deletions of three (1212del3) and nine bp (613del9), respectively; and one insertion of a single base (1094insl). The 304dell deletion at codon 102 in exon 2 caused a frameshift mutation and premature termination of the polypeptide chain. The $777 \mathrm{del}$ mutation resulted in a frameshift in codon 259 in exon 5 with premature termination of the polypeptide chain at amino acid 268. The 1077 dell mutation caused a frameshift in codon 359 , the incorporation of an abnormal sequence of 30 amino acids, and resulted in premature termination of the polypeptide at residue 390 . The dinucleotide deletions included the 1057 del 2 mutation, which caused a frameshift in codon 353 and termination of the polypeptide at residue 373 of exon 7 , and the 1074 del 2 mutation, which resulted in a frameshift in codon 358 and premature termination at residue 373 . The predicted amino acid sequences of these two frameshift mutations were totally different from the normal sequence and different from each other. The insertion mutation (1094insl) predicted a frameshift at codon 365 and premature termination of the polypeptide at codon 374 .

There were two in-frame deletions of three and nine bases, respectively. The $1212 \mathrm{del} 3 \mathrm{mu}-$ tation deleted the trinucleotide AAG of the normal sequence (TTA AGA AGT CAC) which predicted the in-frame deletion of an arginine at position 404 . The 613 del9 mutation involved the

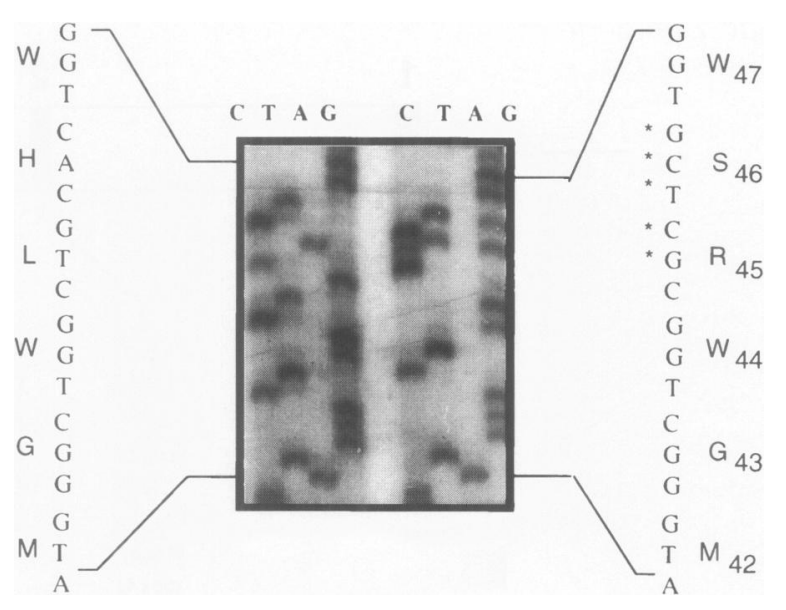

FIG. 2. Nucleotide sequence for the exon 1 complex mutation

One-letter amino acid abbreviations are used and codon numbers are indicated. The deleted nucleotides are indicated by the asterisks and the amino acid substitutions are shown on the right.

in-frame deletion of amino acids P205, L206, and Y207. The breakpoints of this deletion were flanked by the tetranucleotide repeat GTGG in this highly repetitive region, suggesting that the rearrangement may have resulted from slipped mispairing of the repeats during DNA replication (GAG TGG CCT CTT TAT ATG TGG CCC).

\section{$\alpha$-Galactosidase A Complex Mutations}

Two complex mutations were identified in exons 1 and 2 of the $\alpha$-Gal A gene. The exon 1 rearrangement resulted from a series of sequence alterations occurring between the trinucleotide short direct repeat CTGG (normal sequence = CTGG CTGCA CTGG; mutant sequence = CTGG CGCTC GTGG). A five-base sequence between the short direct repeats was changed from TGCAC to GCTCG, resulting in the in-frame replacement of leucine and histidine at codons 45 and 46 by arginine and serine (L45R, H46S). The complex rearrangement in exon 2 involved a series of three small deletions in a 13-bp region (G GCA GAG CTC ATG) from nucleotide 216 to 228 (Fig. 2). There were a total of four bases deleted and therefore a frameshift was predicted at codon 72 , resulting in premature termination of the polypeptide at residue 120 . The relatively high GC content of this region was notable, as well as the presence of dinucleotide repeats GC and AG, which each occurred twice in this re- 
gion. In addition, the deletion of TG occurred at an inverted repeat of TG GT.

\section{DISCUSSION}

In this study, 35 mutations in the $\alpha$-Gal A gene were detected in 42 unrelated families with Fabry disease. Only seven families had previously identified mutations (R49S, P205T, W226X, D313Y, and R227X occurred in one family each, while Q157X occurred in two families), further supporting the fact that almost all of the $\alpha$-Gal A mutations causing Fabry disease are family-specific. Notably, two of these lesions occurred at CpG dinucleotides (R49S and R227X), which are known hot-spots for mutation (20). Of the 35 newly identified lesions, $61.7 \%$ were missense mutations, $3 \%$ were splice site defects, $6 \%$ were nonsense mutations, $23.5 \%$ were small insertions or deletions, and $6 \%$ were complex mutations. No large deletions were detected. All of these mutations were confirmed in genomic DNA from other heterozygous or hemizygous family members identified by enzyme assay (data not shown). Moreover, sequencing of the entire coding region and flanking intronic sequences revealed no other alterations.

All the mutations caused the classic disease manifestations, with the exception of I91T and F113L, which were identified in two unrelated cardiac variants who were diagnosed at 56 and 45 years of age, and had residual (less than 10\%) of normal plasma $\alpha$-Gal A activity. These patients were essentially asymptomatic during most of their lives and did not experience the early classical signs and symptoms that include acroparesthesias, angiokeratoma, corneal and lenticular opacities, and hypohidrosis. The 56-year-old patient had mild proteinuria and LVH, whereas the 45-year-old patient had no renal or cardiac findings, but was ascertained to have the disease after his 71-year-old mother was diagnosed as a carrier with cardiac manifestations. Similarly, the A97V mutation was identified in an asymptomatic 39-year-old male who was diagnosed by enzymatic assay after his mother's renal biopsy showed that she had renal insufficiency and lysosomal storage consistent with Fabry disease. This male patient had no proteinuria, corneal involvement, or cardiac findings. Serial clinical evaluations of these and other cardiac variants will help delineate the natural history of the mild form of this disease. Finally, A143T was identified in a 2-month-old infant who was serendipi- tously found to have deficient $\alpha$-Gal A activity and had no family history of Fabry disease. His mother was subsequently found to have a plasma $\alpha$-Gal A activity in the carrier range (3.7 $\mathrm{U} / \mathrm{ml}$; mean normal control 10.8 ) and was heterozygous for the Al43T mutation. There were no known affected males in this family, making genetic counseling and possible predictions of disease severity difficult.

Of the insertions and deletions, one each occurred in exons 2,4 , and 5 , while four were in exon 7 . That the majority of the small gene rearrangements occurred in exon 7 is consistent with previous reports of seven other unique rearrangements in this region, indicating that exon 7 is highly susceptible to gene rearrangements. Four of the eight gene rearrangements described here occurred within 13 codons of each other, with two occurring in adjacent codons. This clustering of rearrangements indicated that a "hotspot" may exist in the region of codons 353-365 in exon 7. Analysis of this region revealed the presence of the dinucleotide repeats AT and GA, each of which occurred four times in the region. The presence of these repeats suggests a functional role in the generation of these rearrangements, which possibly result from misalignment of the repeats during DNA synthesis at the replication fork. Previously, an additional "hot-spot" for deletions was identified in exon 2 from codons 111-122 (14).

Insights into the structure-function relations of specific $\alpha$-Gal A amino acids and domains can be gained by noting the position of the point mutations causing classic or variant Fabry disease and their relative conservation in the 20 homologues encoding $\alpha$-Gal A or the evolutionarily related enzyme $\alpha$-N-acetylgalactosaminidase (also known as $\alpha$-Gal B) (21) currently recorded in the GenBank database. For example, the two mutations involving cysteine residues ( $\mathrm{C} 94 \mathrm{Y}$ and C202Y) were highly conserved in $85 \%$ and $90 \%$ of the sequences analyzed, respectively, which indicates their probable importance in the formation of intramolecular disulfide bridges in the enzyme polypeptide. It is noteworthy that seven of the missense mutations (Y86C, L89P, I91T, D92Y, C94Y, R100T, and F113L) occurred in exon 2 , in a highly conserved region presumed to contain residues in the active site (22). Interestingly, six of the seven exon 2 missense mutations were highly conserved in the $20 \alpha$-Gal A and $\alpha$-gal B sequences examined (ranging from $80 \%$ to $95 \%$ conserved), with the divergence occurring in the bacterial sequences. The excep- 
tion is the L89P mutation, which was present only in human $\alpha$-Gal A and $\alpha$-Gal B, and mouse $\alpha$-Gal A sequences. Moreover, the mutations in the two cardiac variants were also located in exon 2 and occurred at moderately to highly conserved residues (I91T, 80\%; F113L, 85\%); this further demonstrates the functional importance of this region.

Other mutations that occurred at highly conserved $(>75 \%)$ residues were Y134S, G138R, S148R, D170V, N263S, and W287C, which indicates their importance in the activity and/or stability of the enzyme. Certain mutations occurred in positions that were conserved residues only in mammalian $\alpha$-Gal A sequences (e.g., A31V, H46R, A97V, and L89P). Presumably, substitutions occurring at less essential residues would be neutral or may retain sufficient enzymatic function to be clinically insignificant.

Both nonsense mutations Q386X and W399X occurred in classically affected patients in exon 7 toward the end of the 429 amino acid sequence, indicating that the carboxy-terminal 44 and 30 residues are critical for enzyme function. In support of this concept, one nonsense mutation (E398X) and one in-frame deletion (1208del3) in this region have been previously reported $(14,15)$. Importantly, the nonsense mutations occurred at residues with the lowest degree of conservation noted for the mutations reported in this series $(4 / 20$ and $2 / 20$ for Q386X and W399X, respectively). Another notable mutation was MlT, in which the translation initiation codon was substituted with a threonine. The next methionine was at codon 42 , which, if utilized, would have deleted the entire 31-residue signal peptide, which in turn would presumably preclude targeting to the endoplasmic reticulum.

Interestingly, with the identification of two additional complex mutations in exons 1 and 2 , four complex mutations have now been identified in the $\alpha$-Gal A gene. Previously, complex mutations were identified in several genes, including the $L D L$ receptor gene (23), the HPRT gene (24), and the serum cholinesterase gene (25). Various mechanisms have been proposed for the generation of these mutations, including gene conversion between evolutionarily related sequences (26), or misalignment of "quasi-palindromic" sequences during DNA replication and the subsequent deletion or insertion of bases that might serve to stabilize a hairpin loop (27). The region of the exon 1 complex mutation forms a hairpin loop structure that would be destabilized with the introduction of the five-base alteration.
Analyses of the $\alpha$-Gal A and $\alpha$-Gal B sequences in exons 1 and 2 do not support gene conversion as the mechanism for generation of these complex mutations.

The identification of these 35 mutations further documents the remarkable genetic heterogeneity of the $\alpha$-Gal A lesions causing Fabry disease. Of the mutations identified to date, the majority $(67 \%)$ have been missense mutations, whereas nonsense $(10 \%)$, splicing $(4 \%)$, and small and large gene rearrangements $(19 \%)$ individually are less frequent occurrences $(14,28)$. Mutation analysis is important for each Fabry family, as the detection of these mutations permits precise diagnosis of the heterozygous carriers of this X-linked recessive gene and provides the ability to perform molecular prenatal diagnosis. In addition, the future characterization of these mutations by crystallographic studies may provide useful structure-function information and insights for genotype/phenotype correlations.

\section{ACKNOWLEDGMENTS}

The authors wish to thank Sheri Mural for assistance in DNA sequencing. We also thank the following physicians for referral of patients and/or diagnostic specimens: Drs. G. Wilson, $M$. Iamethi, P. Aula, E. K. M. Smith, C. Harris, N. Doyle, L. Clarke, A. Bottani, L. Workman, I. R. C. Sparrow, K. MacDermott, G. Hogansson, P. Slee, B. Kramer, G. Grabowski, J. Graf, B. Wolf, A. Skarbonk, and L. Emtestam. This work was supported in part by grants from the National Institutes of Health including a Merit Award (R37 DK34045), a grant (MO1 RR00071) for the Mount Sinai General Clinical Research Center from the National Center of Research Resources, a grant (P30 HD28822) for the Mount Sinai Child Health Research Center, and a grant from the American Heart Association (New York City affiliate).

\section{REFERENCES}

1. Desnick RJ, Ioannou YA, Eng CM. (1995) $\alpha$-Galactosidase A deficiency: Fabry disease. In: Scriver CR, Beaudet AL, Sly WS, Valle D (eds). The Metabolic and Molecular Bases of Inherited Disease, 7th ed. McGraw-Hill, New York, pp. 2741-2784.

2. Nakao S, Takenaka T, Maeda M, Kodama C, 
Tanaka A, Tahara M, Yoshida A, Kuriyama M, Hayashibe H, Sakuraba H, et al. (1995) An atypical variant of Fabry's disease in men with left ventricular hypertrophy. $N$. Engl. J. Med. 333: 288-293.

3. Bishop DF, Calhoun DH, Bernstein HS, Hantzopoulos P, Quinn M, Desnick RJ. (1986) Human $\alpha$-galactosidase A: Nucleotide sequence of a cDNA clone encoding the mature enzyme. Proc. Natl. Acad. Sci. U.S.A. 83: 4859-4863.

4. Bishop DF, Kornreich R, Desnick RJ. (1988) Structural organization of the $\alpha$-galactosidase A gene: Further evidence for the absence of a 3' untranslated region. Proc. Natl. Acad. Sci. U.S.A. 85: 3903-3907.

5. Bishop DF, Kornreich R, Eng CM, Ioannou YA, Fitzmaurice TF, Desnick RJ. (1988) Human $\alpha$-galactosidase: Characterization and eukaryotic expression of the full-length cDNA and structural organization of the gene. In: Salvayre R, Douste-Blazy L, Gatt S (eds). Lipid Storage Disorders. Plenum, New York, pp. 809-822.

6. Kornreich R, Desnick RJ, Bishop DF. (1989) Nucleotide sequence of the human $\alpha$-galactosidase A gene. Nucl. Acids Res. 17: 33013302.

7. Hostikka SL, Eddy RL, Byers MG, Hoyhtya M, Shows TB, Tryggvason K. (1990) Identification of a distinct type IV collagen $\alpha$-chain with restricted kidney distribution and assignment of its gene to the locus of X-linked Alport syndrome. Proc. Natl. Acad. Sci. U.S.A. 87: 1606-1610.

8. Barker DF, Hostikka SL, Zhou J, Chow LT, Oliphant AR, Gerken SC, Gregory MC, Skolnick $\mathrm{MH}$, Atkin $\mathrm{CL}$, Tryggvason $\mathrm{K}$. (1990) Identification of mutations in the COL4A5 gene in Alport syndrome. Science 248: 1224-1227.

9. Vetrie D, Vorechovsky I, Sideras P, Holland J, Davies A, Flinter F, Hammarström L, Kinnon C, Levinsky R, Bobrow M, Smith CIE, Bentley DR. (1993) The gene involved in $\mathrm{X}$-linked agammaglobulinemia is a member of the $s r c$ family of protein-tyrosine kinases. Nature 361: 226-233.

10. Caggana M, Ashley GA, Desnick RJ, Eng CM. (1997) Fabry disease: Molecular carrier detection and prenatal diagnosis by analysis of closely linked polymorphisms at xq22.1. Am. J. Med. Genet. (in press).

11. Eng CM, Desnick RJ. (1994) Molecular basis of Fabry disease: Mutations and polymor- phisms in the human $\alpha$-galactosidase A gene. Hum. Mutat. 3: 103-111.

12. von Scheidt $W$, Eng CM, Fitzmaurice $T F$, Erdmann E, Hübner G, Olsen EGJ, Christomanou H, Kandolf R, Bishop DF, Desnick RJ. (1991) An atypical variant of Fabry's disease confined to manifestations of the heart. N. Engl. J. Med. 324: 395-399.

13. Sakuraba H, Oshima A, Fukuhara Y, et al. (1990) Identification of point mutations in the $\alpha$-galactosidase A gene in classical and atypical hemizygotes with Fabry disease. Am. J. Hum. Genet. 47: 784-789.

14. Eng CM, Niehaus DJ, Enriquez A, Burgert TS, Ludman MD, Desnick RJ. (1994) Fabry disease: Twenty-three mutations including sense and antisense $\mathrm{CpG}$ alterations and identification of a deletional hot-spot in the $\alpha$-galactosidase A gene. Hum. Molec. Genet. 3: 1795-1799.

15. Eng CM, Resnick-Silverman LA, Niehaus DJ, Astrin KH, Desnick RJ. (1993) Nature and frequency of mutations in the $\alpha$-galactosidase A gene causing Fabry disease. Am. J. Hum. Genet. 53: 1186-1197.

16. Desnick RJ, Allen KY, Desnick SJ, Raman MK, Bernlohr RW, Krivit W. (1973) Fabry disease: Enzymatic diagnosis of hemizygotes and heterozygotes: $\boldsymbol{\alpha}$-Galactosidase activities in plasma, serum, urine and leukocytes. J. Lab. Clin. Med. 81: 157-171.

17. Bernstein HS, Bishop DF, Astrin KH, Kornreich R, Eng CM, Sakuraba $H$, Desnick RJ. (1989) Fabry disease: Six gene rearrangements and an exonic point mutation in the $\alpha$-galactosidase gene. J. Clin. Invest. 83: 1390-1399.

18. Sambrook J, Fritsch EF, Maniatis T. (1989). Molecular Cloning: A Laboratory Manual, 2nd ed. Cold Spring Harbor Laboratory, Cold Spring Harbor, New York.

19. Kornreich R, Desnick RJ. (1993) Fabry disease: Detection of gene rearrangements in the human $\alpha$-galactosidase A gene by multiplex PCR amplification. Hum. Mutat. 2: 108-111.

20. Cooper DN, Youssoufian H. (1988) The CpG dinucleotide and human genetic disease. Hum. Genet. 78: 151-155.

21. Wang AM, Bishop DF, Desnick RJ. (1990) Human $\alpha$ - $\mathrm{N}$-acetylgalactosaminidase: Molecular cloning, nucleotide sequence, and expression of full length cDNA. Homology with human $\alpha$-Gal A suggests evolution 
from a common ancestral gene. J. Biol. Chem. 265: 21859-21866.

22. Zeidner KM, Ioannou YA, Desnick, RJ. (1993) Human $\alpha$-Gal A active site residue: Determination by homology, mutagenesis, and transient expression. Am. J. Hum. Genet. 53: $1682 \mathrm{~A}$.

23. Yamakawa-Kobayashi $\mathrm{K}$, Kobayashi $\mathrm{T}$, Yanagi H, Shimakura Y, Satoh J, Hamaguchi H. (1994) A novel complex mutation in the LDL receptor gene probably caused by the simultaneous occurrence of deletion and insertion in the same region. Hum. Genet. 93: 625-628.

24. Gibbs RA, Nguyen P-N, McBride LJ, Koepf SM, Caskey CT. (1989) Identification of mutations leading to the Lesch-Nyhan syndrome by automated direct DNA sequencing of in vitro amplified cDNA. Proc. Natl. Acad. Sci. U.S.A. 86: 1919-1923.

25. Nogueira CP, McGuire MC, Graeser C, Bar- tels CF, Arpagaus $M$, Van der Spek AFL, Lightstone H, Lockridge O, La Du BN. (1990) Identification of a frameshift mutation responsible for the silent phenotype of human serum cholinesterase, Gly 117 (GGT $\rightarrow$ GGAG). Am. J. Hum. Genet. 46: 934-942.

26. Pease LR, Horton RM, Pullen JK, Yun TJ. (1993) Unusual mutation clusters provide insight into class I gene conversion mechanisms. Mol. Cell Biol. 13: 4374-4381.

27. Cooper DN, Krawczak M. (1991) Mechanisms of insertional mutagenesis in human genes causing genetic disease. Hum. Genet. 87: 409-415.

28. Davies JP, Eng CM, Hill JA, Malcolm S, MacDermot S, Winchester B, Desnick RJ. (1996) Fabry disease: $14 \alpha$-galactosidase A mutations in unrelated families from the United Kingdom and Europe. Eur. J. Hum. Genet. 4: 219-224.

Communicated by E. Beutler. Accepted December 3, 1996. 